\title{
Call for Papers for a special i-JAC issue devoted to cross-cultural communication and diversity in corporate e-learning.
}

\author{
Guest Editors: B. Kerr ${ }^{1}$, C. Merl ${ }^{2}$ \\ ${ }^{1}$ Concordia University, Montreal, Canada \\ ${ }^{2}$ TalkShop/2CG, Vienna, Austria
}

\begin{abstract}
We are pleased to announce the first call for papers for a special issue devoted to cross-cultural communication and diversity in corporate e-learning.
\end{abstract}

Original contributions, not currently under review or accepted by another journal, are solicited in relevant areas including (but not limited to) the following:

- Cultural diversity in corporate e-learning

- Internationalization and localization of course content

- Cultural studies, information society and digital culture

- Cultural technology and info-communication industry

- Cultural economics, cross-cultural marketing and crosscultural communication in corporate e-learning

- Trends and best practices in global corporate e-learning

- The role of visuals and non verbal practices that can help enhance cross-cultural communication in global business environments

- Practical tools for HR managers to take back to their organisations to optimise diversity for better performance in global business environments.
Prospective authors are asked to look at the iJAC submission guidelines

http://online-journals.org/ijac/about/submissions\#authorGuidelines and use the iJAC template available on the submission page.

\section{IMPORTANT DATES:}

Full paper due for review: 15 June, 2010

Notification of acceptance (with any requested changes): 25 September, 2010 